\title{
Electroconvulsive therapy for a psychotic adolescent during the first trimester of pregnancy: case study
}

\author{
Eletroconvulsoterapia para uma adolescente psicótica durante o primeiro \\ trimestre de gestação: estudo de caso
}

\begin{abstract}
Psychiatric disorders may be present during pregnancy, imposing a burden on the patient, their family and society. ${ }^{1}$ The emergence of psychiatric disturbances during this vulnerable phase may be caused by exacerbation of a preexisting disorder or may be a new condition related to pregnancy itself. ${ }^{1}$ Treating a pregnant patient is a challenge for the psychiatric team because of the possibility of adverse effects on the patient and the fetus. In this scenario, electroconvulsive therapy (ECT) has been identified as a relevant therapeutic strategy for pregnant patients with severe mental illness. ${ }^{2}$ Recently, a review of 33 cases of mental disorders during pregnancy treated with ECT between 1941 and 2007 concluded that ECT is an effective treatment for severe mental illness during pregnancy and that there is low risk to the fetus and the mother. ${ }^{3}$ ECT has also been increasingly used safely with adolescents. ${ }^{4}$ A study of the long term effects of ECT on cognitive functions in adolescents did not detect any measurable anterograde memory deficit 3.5 years after ECT treatment. Additionally, no differences were detected between an ECT group and a comparison group in Mini Mental State Examination (MMSE) scores, scores for the attention section of the Weschler Memory Scale - Revised or California Verbal Learning Test scores. ${ }^{5}$ ECT treatment did not affect adolescents' school or social functioning. ${ }^{6}$

We hereby report a case of a 16 year-old adolescent at 3 weeks gestation who had progressively developed psychotic symptoms over the previous 9 months, including social isolation, irritability, insomnia, delusional speech and visual and auditory hallucinations. Indeed, the patient met the criteria for schizophrenia set out in the Diagnostic and Statistical Manual of Mental Disorders, 5th edition (DSM-5). Symptom assessment
\end{abstract}

was conducted using clinical interviews, rather than structured assessment scales. Antipsychotic treatment was initiated with haloperidol up to a daily dosage of $7.5 \mathrm{mg}$. No clinical improvement was observed after 6 weeks. Further treatment with ECT was discussed because the psychotic scenario remained unchanged and the pregnancy limited the possibility of further pharmacological approaches. The patient underwent 15 ECT sessions. These sessions took place over a 5 weekperiod ( 3 sessions per week) and were accompanied by a progressive improvement in psychotic features. The procedure was authorized by a family member using a previously IRB-approved informed consent form. Haloperidol was continued during follow-up. Maintenance therapy was administered for the next 3 months, with 2 sessions a week during the first 60 days and weekly sessions thereafter. During follow-up the patient attended regular medical appointments and exhibited clinical stability, with no psychotic features after 3 months, when ECT was discontinued due to clinical stability. The patient exhibited progressive amelioration of both negative and positive symptoms. Symptoms had undergone clinical remission within 3 months of follow-up.

ECT has been shown to be safe during all trimesters of pregnancy, but medical attention with adequate life support strategies is recommended. Certain safety issues related to the patient should be addressed, such as the increased risk of aspiration of regurgitated gastric contents during ECT because of longer gastric emptying time among pregnant women and the increased risk of aortocaval compression during advanced pregnancy. With regard to the fetus, a retrospective study of 300 cases of ECT administered during pregnancy found that the most 
common complication was fetal cardiac arrhythmia $(1.6 \%)$. These disturbances were, however, transient and self-limited. ${ }^{7}$

Physicians should also be aware of general physiological aspects related to pregnancy and must judge each case individually to reduce potential risks. We report a case of an adolescent with psychosis who successfully underwent an ECT protocol with clinical amelioration of her symptoms. In this case, ECT proved to be an effective and safe strategy.

\section{Pedro Shiozawa, Alisson Trevizol, Rafael R. Bernardon, Quirino Cordeiro}

Laboratory of Neuromodulation, Department of Psychiatry, Irmandade da Santa Casa de Misericórdia de São Paulo, São Paulo, SP, Brazil.

\section{References}

1. Gressier F, Letranchant A, Hardy P. [Post-partum psychosis]. Rev Prat. 2015;65:232-4.

2. Pompili M, Dominici G, Giordano G, Longo L, Serafini G, Lester D, et al. Electroconvulsive treatment during pregnancy: a systematic review. Expert Rev Neurother. 2014;14:1377-90.

3. Bulbul F, Copoglu US, Alpak G, Unal A, Demir B, Tastan MF, et al. Electroconvulsive therapy in pregnant patients. Gen Hosp Psychiatry. 2013;35:636-9.

4. Jacob P, Gogi PK, Srinath S, Thirthalli J, Girimaji S, Seshadri S, et al. Review of electroconvulsive therapy practice from a tertiary Child and Adolescent Psychiatry Centre. Asian J Psychiatry. 2014;12:95-9.

5. Taieb O, Cohen D, Mazet P, Flament M. Adolescents' experiences with ECT. J Am Acad Child Adolesc Psychiatry. 2000;39:943-4.

6. Taieb O, Flament MF, Chevret S, Jeammet $\mathrm{P}$, Allilaire JF, Mazet $P$, et al. Clinical relevance of electroconvulsive therapy (ECT) in adolescents with severe mood disorder: evidence from a followup study. Eur Psychiatry. 2002;17:206-12.

7. Miller LJ. Use of electroconvulsive therapy during pregnancy. Hosp Community Psychiatry. 1994;45:444-50.

\section{Correspondence:}

Pedro Shiozawa

Department of Psychiatry, Irmandade da Santa Casa de Misericórdia de São Paulo

Rua Major Maragliano, 241, Vila Mariana

04017-030 - São Paulo, SP - Brazil

Tel.: +55 (11) 3466.2100

E-mail: pedroshiozawa@gmail.com

Financial support: none.

Submitted May 25 2015, accepted for publication Jul 282015. No conflicts of interest declared concerning the publication of this letter.

Suggested citation: Shiozawa P, Trevizol A, Bernardon RR, Cordeiro Q. Electroconvulsive therapy for a psychotic adolescent during the first trimester of pregnancy: case study [letter]. Trends Psychiatry Psychother. 2015;37(3):166-167. http:// dx.doi.org/10.1590/2237-6089-2014-0058

Trends Psychiatry Psychother. 2015;37(3) - 167 